\title{
Régulation du récepteur de l'inositol 1,4,5-triphosphate
}

Le contrôle de l'homéostasie du $\mathrm{Ca}^{2+}$ représente une activité fondamentale des cellules eucaryotes et est essentiel au rôle de messager intracellulaire du $\mathrm{Ca}^{2+}$. La concentration de $\mathrm{Ca}^{2+}$ cytosolique $\left[\mathrm{Ca}^{2+}\right]_{i}$ maintenue à un niveau très bas dans une cellule au repos (50-200 nM) peut augmenter rapidement après une stimulation hormonale [1]. La mobilisation du $\mathrm{Ca}^{2+}$ accumulé dans des réservoirs intracellulaires est un événement primordial dans la transduction du signal hormonal et la plupart des éléments moléculaires nécessaires pour constituer des organites capables de stocker et libérer rapidement le $\mathrm{Ca}^{2+}$ ont été identifiés au cours de ces dernières années [2]. Il est admis qu'au moins trois types de protéines sont nécessaires pour constituer un réservoir calcique fonctionnel : une $\mathrm{Ca}^{2+}$-ATPase responsable du transport du $\mathrm{Ca}^{2+}$ à travers la membrane; une ou plusieurs protéines ayant une grande capacité de liaison du $\mathrm{Ca}^{2+}$, localisées à l'intérieur du réservoir; et enfin un canal calcique capable de libérer rapidement le $\mathrm{Ca}^{2+}$ après activation. Le canal calcique responsable de la mobilisation du $\mathrm{Ca}^{2+}$ intracellulaire des cellules non musculaires est activé par l'inositol 1,4,5-triphosphate (InsP3) produit de l'hydrolyse du phosphatidylinositol 4,5-bisphosphate membranaire [3]. Le récepteur de l'InsP3, particulièrement abondant dans les neurones de Purkinje du cervelet, a été identifié ; son ADNc a été cloné et caractérisé. Ce récepteur est constitué de quatre sous-unités identiques ayant chacune un poids moléculaire estimé à $260 \mathrm{kDa}$. La structure primaire du monomère $\mathrm{m} / \mathrm{s} n^{\circ} 10$, vol. 10 , octolme 94 a été déduite de la séquence de l'ADNc du cervelet de souris ou de rat. Les domaines transmembranaires (probablement six) contenant la partie canal ont été localisés du côté carboxy-terminal et la très large partie de la molécule dirigée vers le cytoplasme est constituée d'un domaine de liaison du côté aminoterminal et d'un domaine intermédiaire de couplage avec le canal calcique [4] (figure 1). Cette structure rappelle particulièrement celle du récepteur de la ryanodine, canal calcique du réticulum sarcoplasmique des fibres musculaires impliqué lui aussi dans la libération de $\mathrm{Ca}^{2+}$ intracellulaire. Plusieurs types de récepteur de l'InsP3 produits par quatre gènes différents ont été identifiés et différentes isoformes du récepteur de type 1 proviennent de l'épissage de deux segments localisés, respectivement, dans le domaine de liaison et le domaine de couplage [5].

Cependant, la diversité moléculaire des éléments impliqués dans l'homéostasie du $\mathrm{Ca}^{2+}$ intracellulaire ne permet pas de rendre compte de l'extraordinaire complexité du développement spatial et temporel de la réponse calcique observée dans les cellules uniques stimulées par un agoniste $[6,7]$. L'observation des fluctuations de la $\left[\mathrm{Ca}^{2+}\right]_{i}$, ou « oscillations calciques", laisse supposer que la libération du $\mathrm{Ca}^{2+}$ par le canal sensible à l'InsP3 peut être soumise à des mécanismes de régulation. La propagation d'une vague calcique à travers le cytoplasme, souvent associée aux oscillations, suggère que les organites intracellulaires impliqués dans la mobilisation du $\mathrm{Ca}^{2+}$ sont très précisément organisés au sein de la cellule. I)es modèles expérimentaux simplifiés ont été utilisés afin de disséquer les étapes impliquées dans le développement des réponses calciques au cours d'une stimulation hormonale. Des cellules dont la membrane plasmique a été spécifiquement perméabilisée, ou des microsomes enrichis en récepteur de l'InsP3, ont permis d'analyser les séquences d'événements impliqués dans les variations de $\left[\mathrm{Ca}^{2+}\right]_{i}$ en aval de la production d'InsP3. Il est ainsi apparu que la libération de $\mathrm{Ca}^{2+}$ par l'InsP3 était particulièrement complexe. La première surprise fut de constater que les faibles concentrations d'InsP3 ne peuvent libérer qu'une fraction du contenu du (des) réservoir(s) sensible(s) à l'InsP3. Cette observation suggère que, soit les réservoirs calciques présentent des sensibilités différentes pour l'InsP3, soit il existe une forme de rétrocontrôle de la libération de $\mathrm{Ca}^{2+}[8]$. Par ailleurs, la sensibilité du canal calcique à l'InsP3 dépend de manière biphasique de la concentration du $\mathrm{Ca}^{2+}$ dans le milieu d'incubation. Aux faibles concentrations $(<0,3 \mu \mathrm{M})$, le $\mathrm{Ca}^{2+}$ agit comme un co-agoniste alors que, aux concentrations plus élevées, il inhibe l'effet de l'InsP3 conduisant ainsi à un rétrocontrôle négatif $[9,10]$. Ces observations suggèrent que les propriétés du récepteurcanal de l'InsP3 dépendent de la présence de régulateurs dans le cytosol. Le récepteur de l'InsP3, cible de nombreuses régulations par les protéine kinases, les nucléotides adényliques, le $\mathrm{pH}$ ou les cations divalents, est susceptible de participer à la complexité du signal calcique. 
Régulation par le $\mathrm{pH}$, le $\mathrm{Mg}^{2+}$ et le
$\mathrm{Ca}^{2+}$

L'étude des caractéristiques de liaison de l'InsP3 à son récepteur dans plusieurs tissus a fait apparaître des divergences importantes. La constante de dissociation varie de 2 à $300 \mathrm{nM}$ selon les tissus et, parfois, au sein d'un même tissu comme le cervelet, on peut trouver des différences d'un facteur 10 selon les études [11]. Cela suggère que le récepteur de l'InsP3 peut présenter des propriétés différentes selon son origine tissulaire, mais aussi selon les conditions expérimentales utilisées. Ainsi, par exemple, la liaison de l'InsP3 à son récepteur augmente en fonction du $\mathrm{pH}$ pour être maximale à $\mathrm{pH} 8,0-8,5$. Cet effet est dû à une augmentation de l'affinité du ligand pour son récepteur. Il est possible que, in vivo, l'alcalinisation du cytosol résultant de la stimulation des cellules par certains facteurs de croissance ou certaines hormones puisse entraîner une facilitation de la libération de $\mathrm{Ca}^{2+}$.

Les cations divalents sont également impliqués dans la régulation du récepteur de l'InsP3. Le $\mathrm{Mg}^{2+}$ entre $10 \mu \mathrm{M}$ et $1 \mathrm{mM}$ inhibe de façon non compétitive la liaison de l'InsP3; cependant, il n'est pas évident que cet effet ait des répercussions physiologiques. Le $\mathrm{Ca}^{2+}$ cytosolique intervient de façon beaucoup plus importante dans la régulation des propriétés du récepteur de l'InsP3. Il a été démontré que le $\mathrm{Ca}^{2+}$ inhibe la liaison de l'InsP3 sur des microsomes préparés à partir de cervelet, la concentration de $\mathrm{Ca}^{2+}$ provoquant la moitié de l'effet maximum étant d'environ $0,3 \mu \mathrm{M}$. Cet effet pourrait rendre compte de l'inhibition par le $\mathrm{Ca}^{2+}$ de la mobilisation du $\mathrm{Ca}^{2+}$ intracellulaire observée dans différents types cellulaires. Le récepteur purifié est insensible au $\mathrm{Ca}^{2+}$ et la sensibilité est restaurée par l'addition d'une protéine membranaire intrinsèque de $15 \mathrm{kDa}$, appelée calmédine. Cette protéine est présente dans les cellules du système nerveux central mais semble peu abondante dans les tissus périphériques [12]. L'inhibition de la libération de $\mathrm{Ca}^{2+}$

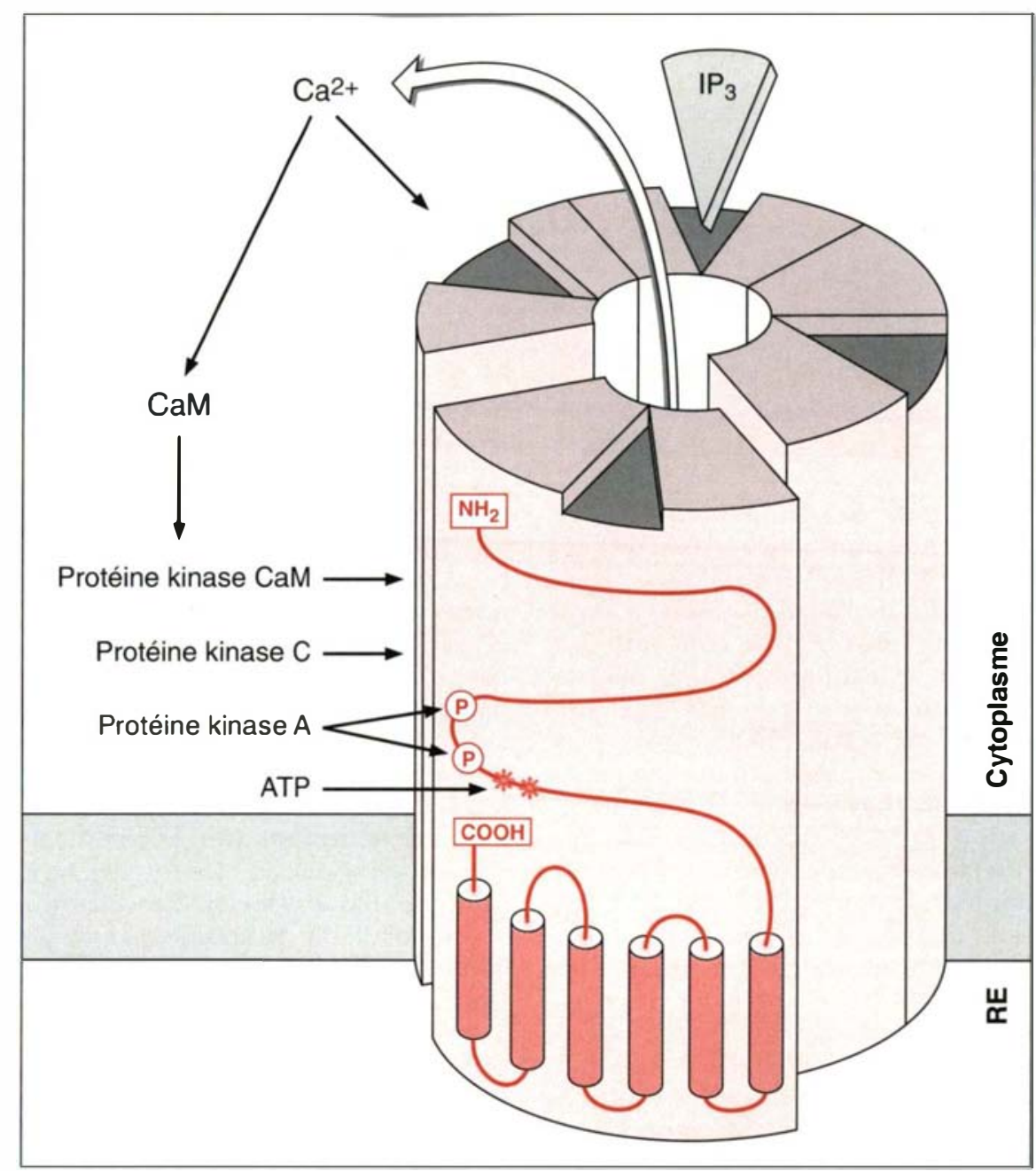

Figure 1. Représentation schématique du récepteur de l'InsP3. Les six domaines transmembranaires sont localisés dans la partie carboxy-terminale, le domaine de liaison est situé dans la partie amino-terminale. Un domaine de régulation contient les sites de phosphorylation $(P)$ par la protéine kinase $A$ et les sites de liaison de l'ATP. Les sites phosphorylés par la protéine kinase $C$ ou la protéine kinase dépendante de la calmoduline (protéine kinase CaM) ne sont pas identifiés. Le récepteur est constitué de quatre sous-unités, contenant chacune un site de liaison pour I'InsP3. Le $\mathrm{Ca}^{2}+$ libéré du réticulum endoplasmique (RE) peut interagir avec la calmoduline (CaM) et entraîner une phosphorylation du récepteur. II peut aussi, par une voie inconnue, modifier les propriétés de liaison du récepteur.

la plupart des cellules ne peut donc pas s'expliquer uniquement par une diminution d'affinité dépendante de la calmédine. De plus, l'inhibition de la liaison de l'InsP3 par le complexe calmédine- $\mathrm{Ca}^{2+}$ ne permet pas d'expliquer le rôle de co-agoniste attribué au $\mathrm{Ca}^{2+}$ dans plusieurs types cellulaires. Un autre mécanisme de régulation par le $\mathrm{Ca}^{2+}$ a été proposé à partir d'expériences effectuées sur des hépatocytes ou des fractions membranaires préparées à partir de foie de rat. Des expériences de liaison et de libération de $\mathrm{Ca}^{2+}$ par l'InsP3 menées en parallèle sur des 


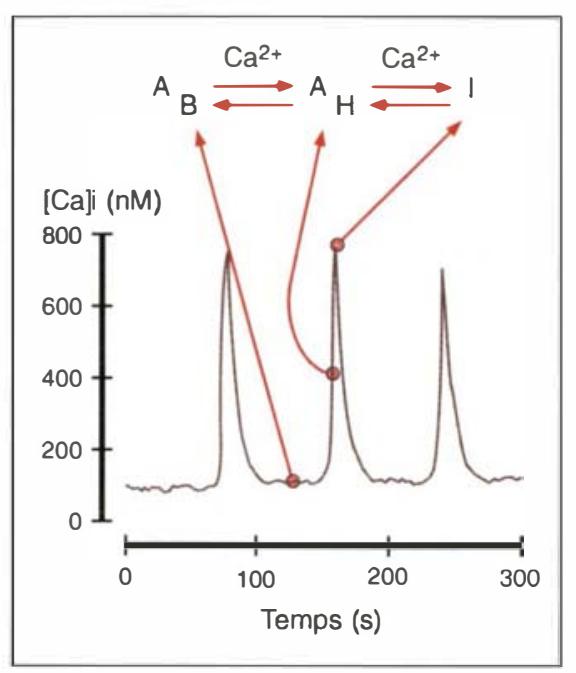

Figure 2. Oscillations de la $\left[\mathrm{Ca}^{2+}\right]$ induites par la noradrénaline dans un hépatocyte isolé. Lorsque la $\left[\mathrm{Ca}^{2+}\right]_{i}$ est à son niveau de base (100 nM), le récepteur de I'InsP3 est dans un état de basse affinité actif $\left(A_{B}\right)$. En présence d'InsP3, la $\left[\mathrm{Ca}^{2+}\right]_{i}$ augmente et l'affinité de la forme active du récepteur augmente pour donner la forme active de haute affinité $\left(A_{H}\right)$. Lorsque la $\left[\mathrm{Ca}^{2+}\right]_{i}$ dépasse $600 \mathrm{nM}$, le récepteur bascule dans un état inactif (I) ayant une haute affinité pour I'InsP3. La $\left[\mathrm{Ca}^{2+}\right]$ revient alors à son niveau de base.

hépatocytes perméabilisés ont permis de définir deux états du récepteur de l'InsP3 [13]. Un état (I), inactivable, se définit par une forte affinité pour l'InsP3 ( $\mathrm{Kd}=2 \mathrm{nM})$ et par des cinétiques d'association et de dissociation très lentes, incompatibles avec les cinétiques extrêmement rapides de libération du $\mathrm{Ca}^{2+}$ intraréticulaire [14]. Un état activable (A) présente des affinités variables pour l'agoniste (Kd entre 4 et $50 \mathrm{nM}$ ) ayant en commun une cinétique d'association rapide, compatible avec la cinétique de libération du $\mathrm{Ca}^{2+}$. Cette forme A est sensible aux agents réactifs des fonctions thiols comme le thimérosal ou le glutathion oxydé qui augmentent l'affinité du récepteur pour l'InsP3 et sensibilisent la libération de $\mathrm{Ca}^{2+}$ aux faibles concentrations d'InsP3 [15]. Cet effet est retrouvé dans différents tissus, y compris le cervelet, indiquant que la régulation de la forme A concerne plusieurs types cellulaires. Dans le foie, le $\mathrm{Ca}^{2+}$ produit deux effets différents selon sa concentration. Aux faibles concentrations, le $\mathrm{Ca}^{2+}$ reproduit l'effet des réactifs des fonctions thiols et augmente l'affinité de la forme A du récepteur, conduisant ainsi à une sorte de rétrocontrôle positif, ce qui explique l'effet de coagoniste attribué au $\mathrm{Ca}^{2+}$ cytosolique au cours de la libération du $\mathrm{Ca}^{2+}$ intraréticulaire [16]. Les concentrations plus élevées de $\mathrm{Ca}^{2+}$ transforment le récepteur en forme I, conduisant ainsi au rétrocontrôle négatif observé dans les hépatocytes comme dans d'autres cellules [10]. ll faut remarquer que, selon le tissu considéré, différents mécanismes peuvent produire des effets identiques. Ainsi, le rétrocontrôle négatif est dû, dans le cervelet, à une diminution de l'affinité du récepteur provoquée par le complexe calmédine- $\mathrm{Ca}^{2+}$, et dans le foie, à une transformation du récepteur en forme I à forte affinité. Si on ajoute à cela que le rétrocontrôle positif se développe plus rapidement que le rétrocontrôle négatif [17], on comprend que la libération de $\mathrm{Ca}^{2+}$ par l'InsP3 en fonction de la concentration de $\mathrm{Ca}^{2+}$ cytosolique puisse conduire à de nombreuses combinatoires et à des courbes "en cloche" typiques de chaque type cellulaire.

Plusieurs groupes ont recherché une régulation de l'ouverture du canal par le $\mathrm{Ca}^{2+}$ intraréticulaire. Cette hypothèse a été proposée pour expliquer qu'à concentration sous-maximale, l'InsP3 ne libère qu'une partie du $\mathrm{Ca}^{2+}$ contenu dans le réticulum endoplasmique: la baisse de la concentration de $\mathrm{Ca}^{2+}$ intraréticulaire entraînerait une diminution de l'affinité du récepteur pour l'InsP3, donc une dissociation du complexe ligand-récepteur et la fermeture du canal [18]. Cependant, une telle régulation n'a pas pu être mise en évidence expérimentalement, peutêtre parce que la concentration de $\mathrm{Ca}^{2+}$ intraréticulaire est difficilement contrôlable.

Ces régulations du récepteur de l'InsP3 par le $\mathrm{Ca}^{2+}$ peuvent jouer un rôle fondamental au cours du développement de la réponse de la cellule cible à un agoniste. Dans une cellule au repos, la $\left[\mathrm{Ca}^{2+}\right]_{i}$ est faible (environ $10^{-7} \mathrm{M}$ ) et les réservoirs calciques intracellulaires sont remplis. La production d'InsP3 provoque l'ouverture du canal et, par conséquent, une augmentation de la $\left[\mathrm{Ca}^{2+}\right]_{\mathrm{i}}$ : l'affinité du récepteur pour l'InsP3 est augmentée, entraînant l'ouverture d'un plus grand nombre de canaux ; la sortie du $\mathrm{Ca}^{2+}$ intraréticulaire est accélérée et permet la phase de montée très rapide du pic calcique. Au sommet du pic, la concentration de $\mathrm{Ca}^{2+}$ a atteint un niveau suffisant pour que le récepteur de l'InsP3 se transforme en un état inactif, I : le canal se referme et la $\left[\mathrm{Ca}^{2+}\right]_{\mathrm{i}}$ revient à sa concentration de repos grâce à l'activité des pompes calciques (figure 2). L'inactivation du canal pourrait être amplifiée par l'effet de la diminution de la concentration du $\mathrm{Ca}^{2+}$ intraréticulaire. La fréquence des pics serait, dans cette hypothèse, régulée par d'autres paramètres tels que la vitesse de repompage du $\mathrm{Ca}^{2+}$ dans les réservoirs intracellulaires.

Le fait que le $\mathrm{Ca}^{2+}$ soit un co-agoniste de l'InsP3 signifie qu'une augmentation de la concentration de $\mathrm{Ca}^{2+}$ cytosolique peut abaisser le seuil de sensibilité du récepteur pour l'InsP3 et entraîner une libération de $\mathrm{Ca}^{2+}$ sans variation de la concentration d'InsP3. Ainsi, par exemple, les vagues et les oscillations calciques qui se produisent lors de la fécondation des œufs de hamster peuvent être reproduites par l'injection, soit d'InsP3, soit de $\mathrm{Ca}^{2+}$, et bloquées par un anticorps spécifique du récepteur de l'InsP3 [19]. Ce résultat montre que le récepteur de l'InsP3 peut être le support d'un phénomène de $\mathrm{Ca}^{2+}$-induced $\mathrm{Ca}^{2+}$ release, identique à celui qui dépend du récepteur de la ryanodine.

\section{Phosphorylation du récepteur de l'InsP3}

Deux séquences consensus contenant un résidu sérine ont été localisées dans le domaine de régulation du récepteur de type I et des études in vitro ont montré que le récepteur 
de l'InsP3 peut être phosphorylé par la protéine kinase dépendante de l'AMPc (PKA) [4]. Il est intéressant de remarquer que les deux sites de phosphorylation sont situés de part et d'autre d'un segment absent des tissus périphériques et présent uniquement dans les cellules du système nerveux central. La phosphorylation du récepteur de l'InsP3 par la PKA entraîne une diminution de la sensibilité à l'InsP3 dans des microsomes préparés à partir de cervelet [4]. Cependant, nous ne disposons pas de données sur la signification physiologique de cet événement dans les neurones. En revanche, il est reconnu depuis longtemps que l'AMPc potentialise la mobilisation du $\mathrm{Ca}^{2+}$ dans les hépatocytes et les récents progrès permettant la mesure de la $\left[\mathrm{Ca}^{2+}\right]$, dans des hépatocytes uniques ont permis de remarquer que l'AMPc augmente la fréquence des oscillations calciques induites par les agonistes produisant de l'InsP3 [20]. Une étude récente montre que le glucagon, par l'intermédiaire de l'AMPc, entraîne une phosphorylation du récepteur de l'InsP3 hépatocytaire, accompagnée d'une augmentation de la sensibilité à l'InsP3 [21]. Ces résultats, s'ils sont confirmés, suggèrent que le récepteur de l'InsP3 pourrait représenter un point de rencontre de différentes voies de signalisation intracellulaire. D'autres études préliminaires indiquent que le récepteur de l'InsP3 est aussi substrat de la protéine kinase $C$ et de la protéine kinase dépendante du complexe $\mathrm{Ca}^{2+}$-calmoduline. De plus, le récepteur purifié aurait une activité sérine kinase lui permettant à la fois de s'autophosphoryler et de phosphoryler des substrats exogènes sur des résidus sérine [22]. Cependant, la signification physiologique de ces phosphorylations n'est pas encore bien comprise.

\section{Les nucléotides adényliques}

A des concentrations supérieures à $0,5 \mathrm{mM}$, l'ATP agit comme un inhibiteur compétitif de l'InsP3. Cependant, entre 10 et $500 \mu \mathrm{M}$, il augmente la liaison de l'InsP3 à son récepteur, probablement par une effet, été montré que des analogues radiomarqués de l'ATP pouvaient directement se lier au récepteur purifié ; cela est confirmé par l'identification de séquences spécifiques des sites de liaison des nucléotides adényliques dans le domaine de régulation du récepteur cérébelleux [4]. Des études électrophysiologiques effectuées sur le récepteur incorporé dans des membranes artificielles ont montré que l'ATP augmente la fréquence d'ouverture et la conductance du canal calcique [23]. Il est difficile d'associer une signification physiologique à ces effets de l'ATP, à moins d'admettre qu'il puisse y avoir des variations locales de la concentration d'ATP suffisamment amples pour modifier l'affinité du récepteur de l'InsP3. Cela pourrait se produire après l'activation des $\mathrm{Ca}^{2+}$-ATPases lorsque la $\left[\mathrm{Ca}^{2+}\right]_{i}$ est élevée et contribuer ainsi à diminuer localement la concentration d'ATP [4].

\section{Interaction protéine-protéine}

Un certain nombre de régulateurs comme les différentes protéine kinases ou les nucléotides adényliques ont directement pour cible la protéine réceptrice de l'InsP3. Cependant, nous avons vu que la diminution de l'affinité du récepteur cérébelleux en présence de $\mathrm{Ca}^{2+}$ se faisait par l'intermédiaire d'une autre protéine membranaire, la calmédine. Il apparaît de plus en plus évident que d'autres interactions de type protéine-protéine interviennent dans le fonctionnement du récepteur de l'InsP3. L'association du récepteur à des protéines du cytosquelette, en particulier l'ankyrine, a été démontrée, dans une lignée de lymphome $\mathrm{T}$ [24] et dans des membranes de cervelet [25]. L'ankyrine, associée à la spectrine, interagit avec l'actine pour former un squelette qui sous-tend la membrane plasmique de la plupart des cellules de vertébrés. La liaison de l'ankyrine à des vésicules membranaires préparées à partir de lymphome inhibe la liaison de l'InsP3 et la libération de $\mathrm{Ca}^{2+}$, suggérant que cette protéine puisse jouer un rôle régulateur dans la cellule intacte. De plus, l'interaction du récepteur de l'InsP3 avec le cytosquelette pourrait permettre le maintien du réservoir sensible à l'InsP3 dans des régions spécifiques de la cellule. Il semble que certains compartiments calciques entretiennent des relations étroites avec la membrane plasmique pour modifier les échanges ioniques avec le milieu extracellulaire [26].

Enfin, deux molécules de récepteur de l'InsP3 peuvent interagir entre elles par leur large extrémité aminoterminale et former des ponts entre deux membranes de réticulum endoplasmique. Cela entraîne la formation d'empilements de citernes de réticulum endoplasmique, particulièrement évidents dans les cellules de Purkinje du cervelet riches en récepteur de l'InsP3 [27]. Ces ponts, formés de deux récepteurs de l'InsP3 apposés, rappellent les protubérances protéiques, piliers (feet structures) qui relient la membrane du réticulum sarcoplasmique au système $T$ du muscle squelettique en faisant intervenir le récepteur des dihydropyridines et le récepteur de la ryanodine [28]. Ces empilements ne sont pas des structures permanentes dans les cellules de Purkinje mais se formeraient dans certaines conditions physiologiques et participeraient ainsi aux mouvements dynamiques de la membrane du réticulum endoplasmique. Le rôle de ces interactions récepteur-récepteur n'est pas connu mais il est intéressant de remarquer qu'il faut vingt à cinquante fois plus d'InsP3 pour libérer le $\mathrm{Ca}^{2+}$ des neurones de Purkinje que des tissus périphériques [10]. On peut imaginer que l'apposition en tête à tête de deux récepteurs de l'InsP3 diminue leur sensibilité et limite la libération de $\mathrm{Ca}^{2+}$ des empilements de citernes de réticulum endoplasmique.

En conclusion, le récepteur de l'InsP3 peut être la cible de multiples régulations faisant intervenir des ions, des enzymes ou des interactions protéine-protéine qui peuvent participer au développement complexe de la réponse calcique au cours d'une stimulation hormonale. De plus, il semble maintenant possible que ce récepteur participe à l'organisation des organites intracellulaires 


\section{RÉFÉRENCES}

1. Mauger JP, Claret M. Flux de calcium et contrôle de la glycogénolyse hépatique. médecine/sciences $1987 ; 3: 574-81$.

2. Meldolesi J, Madeddu L, Pozzan T. Intracellular $\mathrm{Ca}^{2+}$ storage organelles in nonmuscle cells. Heterogeneity and functional assignment. Biochim Biophys Acta 1990 ; 1055: 130-40.

3. Guillemette G. Messagers intracellulaires produits par la phospholipase C. médecine/sciences $1989 ; 5: 5704$.

4. Ferris CD, Snyder SH. Inositol 1,4,5 trisphosphate-activated calcium channels. Annu Rev Physiol 1992 ; 54 : 469-88.

5. Mikoshiba M. Inositol 1,4,5-trisphoshate receptor. Trends Pharmacol Sci $1993 ; 14: 86-9$.

6. Berridge MJ. Inositol trisphosphate and calcium signalling. Nature $1993 ; 361$ : 315-25.

7. Pavoine C, Pecker F. Lumière sur le calcium. médecine/sciences $1994 ; 10: 397407$.

8. Muallem S, Pandol SJ, Beeker TG Hormone-evoked calcium release from intracellular stores is a quantal process. $J$ Biol Chem 1989 ; 264 : 205-12.

9. Iino M. Biphasic $\mathrm{Ca}^{2+}$ dependence of inositol 1,4,5-trisphosphate-induced Ca release in smooth muscle cells of the guinea pig Taenia caeci. I Gen Physiol 1990 ; 95 : pig T1-22.

10. Ogden DC, Khodakhah K, Carter TD Gray PTA, Capiod T. Mechanisms of intracellular calcium release during hormone and neurotransmitter action investigated with flash photolysis. J Exp Biol $1993 ; 184$ : 105-27.

11. Taylor CW, Richardson A. Structure and function of inositol trisphosphate receptors. Pharmacol Ther 1991 ; 51 : 97-137.

12. Danoff SK, Supattapone S, Snyder SH. Characterization of a membrane protein from brain mediating the inhibition of inositol 1,4,5-trisphosphate receptor binding by calcium. Biochem J $1988 ; 254$ : 701-5.

13. Piétri F, Hilly M, Mauger JP. Calcium mediates the interconversion between 2 states of the liver inositol 1,45-trisphosphate receptor. I Biol Chem 1990 ; 265 : 17478-85.

14. Champeil P, Combettes L, Berthon B, Doucet E, Orlowski S, Claret M. Fast kinetics of calcium release induced by myoinositol trisphosphate in permeabilized rat hepatocytes. J Biol Chem 1989; 264: 1766573.

15. Missiaen L, Taylor CW, Berridge MJ Spontaneous calcium release from inositol trisphosphate-sensitive calcium stores. Nature 1991 ; 352 : 241-4.

16. Hilly M, Piétri-Rouxel F, Coquil JF, Guy $M$, Mauger JP. Thiol reagents increase the affinity of the inositol 1,4,5-trisphosphate receptor. J Biol Chem 1993 ; 268 : 16488-94.

17. Finch EA, Turner TJ, Goldin SM. Calcium as a coagonist of inositol $1,4,5$ - trisphosphate-induced calcium release. Science $1991 ; 252: 443-6$.

18. Irvine RF. "Quantal " $\mathrm{Ca}^{2+}$ release and the control of $\mathrm{Ca}^{2+}$ entry by inositol phosphates, a possible mechanism. FEBS Lett $1990 ; 263$ : 5-9.

19. Miyazaki S, Yuzaki M, Nakada K, el al. Block of $\mathrm{Ca}^{2+}$ wave and $\mathrm{Ca}^{2+}$ oscillation by antibody to the inositol 1,4,5-trisphosphate receptor in fertilized hamster eggs. Science $1992 ; 257: 251-5$

20. Schöfl C, Sanchez-Bueno A, Brabant G, Cobbold PH, Cuthbertson KSR. Frequency and amplitude enhancement of calcium transients by cyclic AMP in hepatocytes. Biochem J 1991 ; 273 : 799-802.

21. Joseph SK, Ryan SV. Phosphorylation of the inositol trisphosphate receptor in isolated rat hepatocytes. J Biol Chem 1993 ; $268: 23059-65$

22. Ferris CD, Cameron AM, Bredt DS, Huganir RL, Snyder SH. Autophosphorylation of inositol 1,4,5-trisphosphate receptors. J Biol Chem 1992 ; 267 : 7036-41.

23. Maeda N, Kawasaki T, Nakade S, et al. Structural and functional characterization of inositol 1,4,5-trisphosphate receptor channel from mouse cerebellum. J Biol Chem 1991 ; 266 : 1109-16.

24. Bourguignon LYW, Jin $\mathrm{H}$, Iida $\mathrm{N}$, Brandt NR, Zhang SH. The involvement of ankyrin in the regulation of inositol $1,4,5$ trisphosphate receptor-mediated $\mathrm{Ca}^{2+}$ release from $\mathrm{Ca}^{2+}$ storage vesicles in mouse T-lymphoma cells. J Biol Chem 1993; 268 : $7290-7$.

25. Joseph SK, Samanta S. Detergent solubility of the inositol trisphosphate receptor in brain membranes. I Biol Chem $1993 ; 268: 6477-86$

26. Rossier MF, Putney JW. The identity of the calcium-storing, inositol $1,4,5$ trisphosphate-sensitive organelle in nonmuscle cells : calciosome, endoplasmic reticulum... or both. Trends Neurosci 1991 ; $14: 310-4$.

27. Takei K, Mignery GA, Mugnaini E, Südhof TC, Decamilli P. Inositol 1,4,5 trisphosphate receptor causes formation of ER cisternal stacks in transfected fibroblasts and in cerebellar Purkinje cells. Neuron $1994 ; 12: 327-42$.

28. Rousseau $\mathrm{E}$, Chabot $\mathrm{H}$. Identification fonctionnelle du canal calcique du réticulum sarcoplasmique. médecine/sciences $1992 ; 8: 950-7$.

Jean-Pierre Mauger

Directeur de recherche à l'Inserm, Inserm U. 274, bâtiment 443, université ParisSud, 91405 Orsay Cedex, France.

\section{TIRÉS A PART}

J.P. Mauger. 